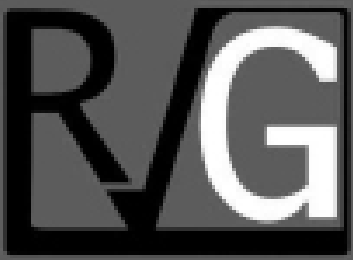

Año 24 No. 88

Octubre - Diciembre 2019

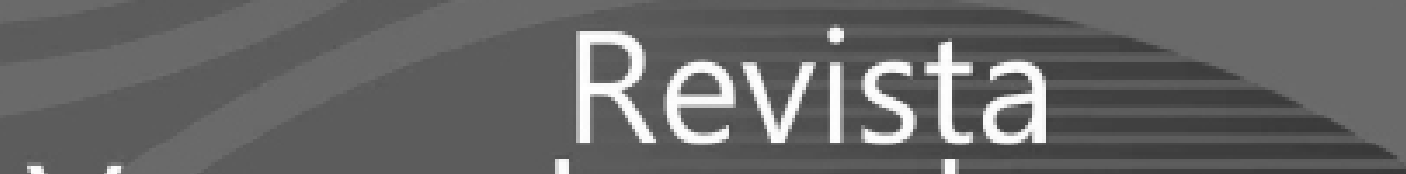

Venezolana de

verencla

UNIVERSIDAD DEL ZULIA (LUZ)

Facultad de Ciencias Económicas y Sociales

Centro de Estudios de la Empresa 


\title{
Contabilidad de gestión ambiental en empresas del sector agroindustrial
}

\author{
Alvarez, Romer ${ }^{1}$ \\ Ferrer, María Alejandra² \\ Galaviz, Betty ${ }^{3}$ \\ Castro, Josefa ${ }^{4}$
}

\section{Resumen}

La contabilidad de gestión ambiental ha tomado un rol importante en relación a la sostenibilidad de las organizaciones. La presente investigación tiene como objetivo analizar la contabilidad de gestión ambiental en empresas del sector agroindustrial del estado Zulia (Venezuela), fundamentándose en Azqueta (2009), Gray y Bebbintong (2006), Mantilla et al (2005), Monroy y Saer (2011). La metodología es descriptiva, no experimental, de campo. La población está conformada por las tres (3) empresas activas productoras de subproducto animal en la elaboración de alimentos balanceados para animales, en el municipio San Francisco del estado Zulia (Venezuela). Los resultados evidencian que las empresas en estudio cuentan con políticas ambientales, contemplan las cuestiones ambientales en sus decisiones de inversión y financiamiento, mantienen registros, valoración, acumulación y revelación de costos, activos y pasivos de tipo medioambiental, y preparan informes sobre materias primas para la toma de decisiones. Sin embargo, carecen de un sistema de contabilidad ambiental formalmente establecido y de indicadores de medición del ámbito ambiental. Se concluye que más allá del cumplimiento de preceptos ambientales formalmente establecidos, la actividad empresarial ha de enfocarse sus esfuerzos al desarrollo sostenible,

Recibido: 31-05-18 Aceptado:18-02-19

1 Profesor titular de la Universidad del Zulia (LUZ-Venezuela), Postdoctorado en Gerencia de las Organizaciones, Doctor en Ciencias Gerenciales, Magister en Gerencia Empresarial, Licenciado en Contaduría Pública, Licenciado en Educación. Email: alvarezromer@hotmail.com. Autor para la correspondencia

** Profesora Titular de LUZ, Doctora en Finanzas de Empresa (UCM-España), Magiste en Gerencia de Empresas Mención Gerencia Financiera (LUZ), Licenciada en Contaduría Pública (LUZ). Email: maferrer99@ yahoo.com

*** Decana y profesora titular de la Universidad Rafael Belloso Chacín (URBE-Venezuela), Doctora en Ciencias Gerenciales, Magister en Gerencia de Empresas Mención Gerencia Industrial, Licenciada en Contaduría Pública. Email: Betty.galaviz@urbe.edu

*** Profesora Agregada de LUZ, Magister en Gerencia Empresarial, Licenciada en Contaduría Pública, Licenciada en Administración. Email: jos2203@hotmail.com 
apoyada en la contabilidad de la gestión ambiental como herramienta para el logro de los objetivos.

Palabras clave: contabilidad de gestión ambiental; activos ambientales; costos ambientales; sector agroindustrial.

\title{
Environmental management accounting in companies of the agro-industrial sector
}

\begin{abstract}
Environmental management accounting has taken an important role in relation to the sustainability of organizations. The purpose of this research is to analyze environmental management accounting in companies in the agro-industrial sector of Zulia state (Venezuela), based on Azqueta (2009), Gray and Bebbintong (2006), Mantilla et al. (2005), Monroy and Saer (2011). The methodology is descriptive, not experimental, field. The population is made up of three (3) companies producing animal by-products in the production of feed for animals, in the municipality of San Francisco in Zulia state (Venezuela). The results show that the companies under study have environmental policies, consider environmental issues in their investment and financing decisions, maintain records, valuation, accumulation and disclosure of environmental costs, assets and liabilities, and prepare reports on raw materials for decision making. However, they lack a formally established environmental accounting system and environmental measurement indicators. It is concluded that beyond the fulfillment of formally established environmental precepts, business activity has to focus its efforts on sustainable development, supported by environmental management accounting as a tool for achieving the goals.
\end{abstract}

Keywords: Environmental management accounting; environmental assets; environmental costs; agroindustrial sector.

\section{Introducción}

En términos generales, las actividades económicas suelen tener impacto negativo en el ambiente y la salud, al tiempo que una economía sin normas causa mermas intolerables en los recursos naturales. En consecuencia, el Estado y el sector empresarial participa en el diseño de políticas ambientales para el sector público y privado. Cualquiera que sea el tipo, incluye las medidas para prevenir o disminuir la contaminación del aire y del agua, así como las que atañen al aprovechamiento de los recursos naturales, y deben funcionar a cualquier nivel espacial y organizacional.

Considerando lo anterior, la Contabilidad de Gestión Ambiental (CGA) tiene la finalidad de medir los 
impactos financieros y no financieros de las acciones de la empresa sobre el medio ambiente cuando realiza su actividad económica, siendo necesaria su aplicación para el desarrollo sostenible de la entidad y de cara a la regulación del calentamiento global.

Las empresas ocasionan daños ambientales con diferentes niveles de complejidad a lo largo de toda su cadena de valor. Por tanto, la estrategia empresarial debe encaminarse al fomento de acciones para incidir en procesos productivos que sean favorablemente compatibles con el medio ambiente. Así, una buena gestión ambiental puede traducirse en valor agregado para la empresa y en una ventaja competitiva, presentándose como impulsor de la modernización de las actividades para acrecentar su productividad.

La implementación de la CGA es una parte medular de esa gestión empresarial para mejorar la identificación, seguimiento y administración de los insumos, energía, desechos e impacto ambiental, así como el proceso contable de los activos, pasivos y costos, de tipo ambiental, a presentar en los estados financieros para la toma de decisiones.

En tal sentido representa un reto para la gerencia financiera de las organizaciones contar con la herramienta de la CGA dentro de sus finanzas para medir con mayor precisión y responsabilidad las cuestiones ambientales que se relacionan con el cumplimiento de las regulaciones, la gestión del negocio, la ecoeficiencia, el valor agregado y la calidad de vida.

Considerando los fundamentos anteriores se tiene que el objetivo del presente estudio es analizar la contabilidad de gestión ambiental en empresas del sector agroindustrial, productoras de subproducto animal en la elaboración de alimentos balanceados para animales, en el municipio San Francisco del estado Zulia (Venezuela). Esta investigación se considera de tipo descriptiva, no experimental, transeccional y de campo, en la que se aplicó un cuestionario de 62 ítems con preguntas cerradas a las unidades informantes constituidas por ocho (8) gerentes y administradores del área de finanzas de las tres (3) empresas activas según la Asociación de Comerciantes e Industriales del estado Zulia (ACIZ) ubicadas en el municipio en referencia: Grasas Bermúdez, C.A., Supergrasos Perijá, C.A. y Prograsa, C.A.

\section{Política ambiental}

Considerada por Azqueta (2009) como el paso más elemental, la adopción de la política ambiental de la empresa consiste en la declaración, por parte de la misma, de su propósito de mejorar su desempeño en el campo del medio ambiente. Esta declaración ha de contenerse en un documento, suscrito por los máximos responsables de la dirección, para garantizar la seriedad del compromiso adquirido, en el que se especifiquen los objetivos que la institución se propone conseguir con respecto al medio ambiente.

Según Gray y Bebbington (2006), el adoptar una política ambiental consiste en tenerla como guía para la acción futura. Por consiguiente, se requieren datos confiables respecto de las interacciones ambientales de la organización, compromisos tan específicos como sea posible y el apoyo de mecanismos para estructurar la política en objetivos específicos. 


\section{Marco legal venezolano}

En el caso de Venezuela, las políticas ambientales se enmarcan en los preceptos enunciados en la Constitución de la República Bolivariana de Venezuela (CRBV) (Asamblea Nacional de la República Bolivariana de Venezuela, 1999) y en la Ley Orgánica del Ambiente (LOA) (Asamblea Nacional de la República Bolivariana de Venezuela, 2006). Dichos preceptos deben ser acatados por todas las empresas para controlar el impacto ambiental, y por consiguiente deben ser contemplados en todos los ámbitos de actuación de la entidad y reflejados en la contabilidad.

El artículo 127 de la CRBV (ANRBV, 1999) dictamina que es un derecho y un deber de cada generación proteger y mantener el ambiente en beneficio de sí misma y del mundo futuro. Toda persona tiene derecho individual y colectivamente a disfrutar de una vida y de un ambiente seguro, sano y ecológicamente equilibrado. El Estado protegerá el ambiente, la diversidad biológica, los recursos genéticos, los procesos ecológicos, los parques nacionales y monumentos naturales $y$ demás áreas de especial importancia ecológica. El genoma de los seres vivos no podrá ser patentado, y la ley que se refiere a los principios bioéticos regulará la materia. Es una obligación fundamental del Estado, con la activa participación de la sociedad, garantizar que la población se desenvuelva en un ambiente libre de contaminación, en donde el aire, el agua, los suelos, las costas, el clima, la capa de ozono, las especies vivas, sean especialmente protegidos, de conformidad con la ley.

Por otra parte, el artículo 2 la LOA (ANRBV, 2006) establece que la gestión ambiental son medidas encaminadas para hacer un diagnóstico, así como inventariar, restaurar, preservar, controlar, custodiar y aprovechar los ecosistemas y demás recursos naturales, en garantía del desarrollo sustentable. Para ello, el artículo 25 de la mencionada ley enuncia que las personas naturales y jurídicas, públicas y privadas, deben planificar sus actividades congruentes con la gestión ambiental.

Para Mantilla et al (2005), lo anterior supone contar con procesos de tecnología limpia que garanticen el uso adecuado de los recursos naturales y del ambiente, no sólo en orden a la legislación nacional e internacional y en coherencia con las políticas sociales y económicas de cada país, sino, además, con técnicas y métodos adecuados de control, medición y evaluación, que hagan posible tanto el seguimiento a procesos y acciones frente al contexto natural, como el aseguramiento de instrumentos para facilitar la orientación del quehacer económico, en procura de bienestar en mejoramiento continuo, definidos por la calidad de vida en un ambiente sano. De allí nace la necesidad de la Contabilidad de Gestión Ambiental.

\section{Contabilidad de gestión ambiental}

Según Mantilla et al (2005), la Contabilidad de Gestión Ambiental (CGA) es el sistema que permite el reconocimiento, organización, valoración, el registro de las condiciones, los cambios en los recursos naturales y del ambiente; el mismo hace posible articular indicadores de evaluación de sostenibilidad del ecosistema en el contexto del desarrollo, vinculando sistemas de información que faciliten el control de las acciones que afectan la condición de la naturaleza y el desarrollo nacional.

Gray y Bebbington (2006) sostienen que la CGA se centra en la cuantificación 
y control de los recursos ambientales, siguiendo un enfoque flujo/entrada-salida basado en una concepción de sistemas referente a las organizaciones que intenta informar sobre los flujos de esos bienes al momento de la rendición de cuentas, proporcionando transparencia a la organización. Se realiza de una manera que facilitará a los trabajadores valorar su uso mediante las entradas y salidas en cantidades financieras y no financieras, permitiéndole además a la organización contribuir con la sostenibilidad mediante actividades que controlan el consumo de los recursos.

Ahora bien, según Van et al (2011), la CGA se centra en los costos ambientales, por ello las decisiones se concentran en: a) la identificación de los costos ambientales escondidos dentro de los costos generales; b) cuantificar ingresos obtenidos por la venta de residuos u otras ganancias; c) cuantificar los costos y beneficios de riesgos del manejo ambiental para sustentar propuestas de mejoramiento; d) aproximar los costos reales de productos y/o procesos, involucrando los costos ambientales; e) apoyar la planificación del manejo ambiental mediante la identificación de puntos críticos; f) mostrar ventajas competitivas en términos económicos de productos menos contaminantes.

\section{Impacto de las cuestiones ambientales sobre los estados contables}

\footnotetext{
Las cuestiones ambientales según Fronti y Wainstein (2010) son definidas como actividades llevadas a cabo voluntariamente, así como también requeridas por contrato o por leyes y regulaciones ambientales, para prevenir, disminuir o remediar el daño causado al medio ambiente, relacionado tanto con
}

la conservación de recursos renovables como con los no renovables.

Dichas cuestiones ambientales podrían tener impacto en los estados financieros, como es el caso de: a) los costos ambientales en que una empresa debería incurrir para recuperar un espacio contaminado por ella; b) casos de contaminación del agua, aire, tierra, entre otros, causados por la empresa y contemplados por las regulaciones ambientales, que obligarán en cualquier momento la pronta reparación de los daños por parte del Estado; c) inversiones en maquinarias y equipos destinadas a la conservación de los recursos naturales y para la reparación de daños ambientales; d) el incumplimiento de las regulaciones ambientales que afectaría la imagen corporativa de la empresa y colocan en riesgo la permanencia de su actividad económica.

En efecto, esas cuestiones ambientales son susceptibles de generar en la contabilidad de las empresas un conjunto reconocible de activos, pasivos y costos de tipo ambiental, que, incluidos en los estados financieros, afectan su estructura financiera, los resultados de la gestión económica, y, por consiguiente, el cumplimiento con las regulaciones ambientales y responsabilidad social.

Según Crespo (2005), se entiende por activos ambientales los elementos incorporados al patrimonio de la empresa para ser utilizados de forma duradera en su actividad, con el objetivo de minimizar el impacto ambiental y la protección y mejora del medio ambiente, incluyendo la eliminación de la contaminación futura.

Un pasivo ambiental es un sitio geográfico contaminado por la liberación de materiales o residuos peligrosos que no fueron tratados oportunamente para impedir su dispersión y, en el momento actual, representan un riesgo para el 
ambiente y la calidad de vida (FUNGLODE, 2013). También surgen como resultado de proyectos $\mathrm{o}$ actividades humanas del pasado, con deterioro progresivo en el tiempo, que puede llegar a afectar la calidad del agua, el suelo, el aire y los ecosistemas. Su condición de pasivos está relacionada con la pérdida del estado previo (un activo ambiental) y, por lo tanto, implica una obligación de reparación o deuda que en algún momento se deberá solventar.

Ahora bien, para Hansen y Mowen (2003), se incurre en los costos ambientales porque existe o puede existir una pobre calidad ambiental. Así, los costos ambientales están relacionados con la creación, detección, remedio, y prevención de la degradación ambiental. Además, contribuyen con la producción de bienes y servicios más útiles con la reducción de impactos ambientales negativos atacando las causas más que las consecuencias, significando esto ser ecoeficiente.

Dichos costos ambientales son las partidas resultantes de las actividades llevadas a cabo voluntariamente, así como también las requeridas por contrato o por leyes y regulaciones ambientales para prevenir, disminuir o remediar el daño causado al medio ambiente, relacionado tanto con la conservación de recursos renovables como no renovables (Phalen y Fronti (2005).

\section{Normas Internacionales de Información Financiera para aspectos ambientales}

La normativa contable para
aspectos ambientales se centra
principalmente en el desglose de
cuestiones o eventos ambientales
que forman parte de los rubros de los
estados financieros y en la incorporación

de notas de los impactos sobre el medio ambiente, situación que tiene sus bases en las regulaciones de las leyes ambientales de cada país y en la responsabilidad social ambiental de la empresa.

Las Normas Internacionales de Información Financiera (NIIF), emitidas por el Consejo de Normas Internacionales de Información Financiera (2011), contempla la valoración y presentación de las diferentes partidas en la contabilidad, siendo importante destacar en este caso las que se encuentran relacionadas con la temática ambiental, ellas son la NIC 1 (IASB, 2011) referente a la presentación de los estados financieros y la NIC 37 (IASB, 2011) relacionada con las provisiones, pasivos y activos contingentes.

De acuerdo con la NIC 1 (IASB, 2011) los estados financieros constituyen una representación estructurada de la situación financiera y del rendimiento financiero de una entidad. Muestran los resultados de la gestión realizada por los administradores con los recursos que les han sido confiados. Para cumplir este objetivo, los estados financieros suministrarán información acerca de los siguientes elementos de una entidad: (a) activos; (b) pasivos; (c) patrimonio; (d) ingresos y gastos; (e) aportaciones de los propietarios y distribuciones a los mismos; y (f) flujos de efectivo.

Por lo tanto, para analizar la situación de una empresa deben incluirse en los estados financieros todas las partidas afectadas a raíz de la toma de decisiones para la actividad económica, incluyendo las de tipo ambiental.

Ahora bien, la NIC 37 (IASB, 2011) establece el tratamiento de las provisiones, pasivos $y$ activos contingentes vinculados con hechos ambientales por parte de las entidades. 
En dicha norma se consideran considera las siguientes definiciones (IASB, 2011):

- Un pasivo es una obligación presente de la entidad, surgida a raíz de sucesos pasados, al vencimiento de la cual, y para cancelarla, la entidad espera desprenderse de recursos que incorporan beneficios económicos.

- Una provisión es un pasivo en el que existe incertidumbre acerca de su cuantía o vencimiento, que para ser contabilizada debe cumplir con unos requisitos, entre ellos, a) la empresa tiene una obligación presente legal o implícita (obligación legal producto de leyes y contratos, u obligación implícita producto de situaciones propias de compromiso por parte de la propia empresa) como resultado de un suceso pasado, b) es probable que la empresa tenga que desprenderse de recursos para cancelarla; y c) pueda hacerse una estimación fiable sobre el importe de dicha obligación.

Un ejemplo de ello es un contrato para la explotación minera en el que la empresa está obligada a reparar los daños ambientales causados. En este caso existe una obligación legal presente según contrato por un hecho de deterioro del ecosistema que será un suceso pasado, además la empresa debe desprenderse de recursos para sufragar la obligación y puede hacerse una estimación fiable de los daños a reparar, por lo tanto, se amerita la realización de una provisión por daños ambientales.

- Un pasivo contingente es: (a) una obligación posible, surgida a raíz de sucesos pasados y cuya existencia ha de ser confirmada sólo porque ocurran o no ocurran uno o más hechos futuros inciertos que no están enteramente bajo el control de la entidad; o (b) una obligación presente, surgida a raíz de sucesos pasados, que no se ha reconocido contablemente porque: (i) no es probable que para satisfacerla se vaya a requerir una salida de recursos; o (ii) el importe de la obligación no pueda ser medido con la suficiente fiabilidad.

Como ejemplo, según Crespo (2005) para un pasivo contingente de tipo ambiental, cuando a una empresa se le presenta una responsabilidad compartida por un problema de contaminación. En este caso la parte de la deuda que se espera que cubran los otros responsables se tratará como un pasivo contingente y solo será objeto de información en las notas de los estados financieros, esto motivado a que existe una baja probabilidad de ocurrencia y además la remota posibilidad de que la empresa deba desprenderse de recursos.

- Un activo contingente es un activo de naturaleza posible, surgido a raíz de sucesos pasados, cuya existencia ha de ser confirmada sólo por la ocurrencia, o en su caso por la no ocurrencia, de uno o más eventos inciertos en el futuro, que no están enteramente bajo el control de la entidad. Por esas características no suelen contabilizarse, solo se expresan mediante la incorporación de información en las notas de los estados financieros en vista de que la entrada de beneficios económicos pudiera ser probable.

Ejemplo de ello sería el caso de una reclamación que realiza una empresa " $A$ " mediante un proceso judicial a otra empresa "B" por 
haber arrojado en sus lagunas una serie de contaminantes que han deteriorado el ecosistema y sus reservas de agua. En este caso existe un suceso pasado calificado como la contaminación de una laguna, el grado de ocurrencia, procedencia del caso, y entrada de beneficios, tiene cierta incertidumbre pues no está enteramente bajo el control de la empresa "A" debido a que depende del resultado de la reclamación con el Estado. Por tanto el reflejo contable para la empresa " $A$ " es una activo contingente sobre el que se debe informar en las notas complementarias a los estados financieros describiendo su situación.

Es importante resaltar que las NIIF (IASB, 2011) inciden en los aspectos contables de las empresas con respecto al reconocimiento, medición y presentación en los estados financieros de partidas de activo, pasivo y costos sobre cuestiones ambientales, por lo que afecta su situación financiera, responsabilidad ambiental y cumplimiento de las regulaciones para tal fin.

\section{Indicadores o medidas ambientales}

Cuando las finanzas empresariales informan a los distintos usuarios sobre los acontecimientos financieros de la empresa, no solo hacen uso de los estados financieros como medio para el análisis y la toma de decisiones, sino que están en capacidad para presentar medidas $o$ indicadores financieros $y$ de gestión necesarios para contribuir a obtener un diagnóstico sobre la situación del negocio. Por lo que resulta evidente para el caso de la presente investigación considerar las medidas o indicadores que las finanzas pueden presentar desde el ámbito ambiental.

Para Pahlen y Fronti (2005), el desarrollo de indicadores para la caracterización de una empresa orientada hacia el medio ambiente es un apoyo importante para la revisión y evaluación de los datos revelados acerca de su situación medioambiental. Esto constituye el incremento de información para la organización y para los sectores externos interesados.

Además, una empresa puede determinar, para un período en particular, el total de sus salidas de fondos con respecto a pérdidas, gastos, costos e inversiones, de tipo ambiental (Pahlen y Fronti, 2005): a) gastos ambientales/ gastos totales; b) pérdidas ambientales/ pérdidas totales, c) costos ambientales/ costos totales; y d) inversiones ambientales/inversiones totales.

Para obtener información sobre el comportamiento ambiental de las empresas, Pahlen y Fronti (2005) consideran que estos indicadores no deben ser analizados en forma aislada, siendo necesario compararlos con indicadores de otras empresas o de otros períodos, y analizar las variaciones.

\section{Agroindustria y contabilidad de gestión ambiental}

El análisis de la contabilidad de gestión ambiental en empresas productoras de subproducto animal en la elaboración de alimentos balanceados para animales ubicadas en el municipio San Francisco del estado Zulia, se centró en la implementación de la política ambiental, las regulaciones ambientales venezolanas, la actividad financiera en el ámbito ambiental, los sistemas de contabilidad ambiental, los 
costos ambientales, y la preparación de información financiera para la toma de decisiones.

Con respecto a la implementación de la política ambiental, las empresas estudiadas cuentan con políticas ambientales, las cuales son formuladas y dadas a conocer a los empleados e interesados. En el diseño de las mismas se toma en cuenta la actividad económica, regulaciones ambientales relacionadas, tipo de negocios y contexto del país. Además, estas políticas son actualizadas periódicamente y su efecto se evalúa al confrontarlas con los resultados de la gestión alcanzada, lo cual ha sido satisfactorio en virtud de que las diferentes gerencias consideran los lineamientos de las políticas ambientales en las actividades realizadas.

El reconocimiento de la política ambiental en estas empresas productoras de subproducto animal es congruente a lo establecido por Azqueta (2009) quien sostiene que la política ambiental permite mejorar el desempeño en el campo del medio ambiente mediante un documento suscrito por los máximos responsables de la dirección, para garantizar la seriedad del compromiso adquirido, en el que se especifiquen los objetivos que la institución se propone conseguir con respecto.

Por otra parte, las políticas ambientales de estas empresas contemplan la actividad financiera como medio para vincular y armonizar la actividad económica con el medio ambiente, de modo que pueda contribuirse con la disminución del impacto ambiental, el cumplimiento de las regulaciones ambientales y con la responsabilidad social corporativa. De manera que, para poner en marcha la contabilidad ambiental se requiere, según Mantilla et al (2015), de un compromiso de la sociedad civil, las empresas y los gobiernos a través de la generación de conciencia que permita un cambio radical implícito en el proceso, y por tanto se deben empezar a implementar políticas que conlleven a este con el fin de minimizar el impacto ambiental producto de las actividades humanas.

En referencia a los preceptos de las regulaciones ambientales venezolanas establecidos en la CRBV (ANRBV, 1999) y en la LOA (ANRBV, 2006), se tiene que en las empresas objeto de estudio se da cumplimiento a las disposiciones de ley. Adicionalmente, en el área de producción y distribución existen listas de verificación extraídas de los diferentes preceptos jurídicos para comprobar su cumplimiento. Sin embargo, dos (2) de las empresas estudiadas fueron objeto de multas por parte de representantes de instituciones del Estado venezolano por el incumplimiento de las normativas ambientales con respecto al higiene del lugar de domicilio.

En cuanto al ejercicio de sus finanzas, las empresas del sector agroindustrial objeto de estudio contemplan en sus actividades de inversión y financiamiento partidas con objetivos ambientales encaminadas a la adquisición de maquinarias, equipos y plantas, que contribuyen a una producción más limpia, como es el caso de filtros electrostáticos y plantas de tratamiento de aguas, adquiridas mediante financiamiento de bancos privados de la región. Dichas inversiones les permiten cumplir con los compromisos establecidos en sus políticas ambientales y con las regulaciones del Estado venezolano, lo cual ha permitido sanear sus espacios y disminuir el impacto ambiental. Por lo que esta situación favorable 
es compatible con lo establecido por Mantilla et al (2005), quienes establecen que es importante contar con procesos de tecnología limpia para garantizar el uso adecuado de los recursos naturales y del ambiente, no sólo en orden a la legislación nacional e internacional y en coherencia con las políticas sociales y económicas de cada país, sino, además, con técnicas y métodos adecuados de control, medición y evaluación.

Si bien estas organizaciones no cuentan con un sistema de contabilidad ambiental formalmente establecido, en la contabilidad financiera se mantienen procesos de registro, acumulación, valoración y revelación, de los activos, pasivos, provisiones y contingencias, por prevención y daños, de tipo ambiental, sin dicha denominación. De esta manera se incorporan de manera parcial los lineamientos de las Normas Internacionales de Información Financiera (IASB, 2011) en cuanto a los elementos de los estados financieros anteriormente mencionados, debido a que para efectos de presentación es fundamental identificar las partidas con la denominación ambiental.

La ausencia de un sistema de CGA dentro de la contabilidad financiera constituye una limitante importante para las empresas, pues, en palabras de Lozano (2016), el apoyo del área contable en la toma de decisiones ambientales será un factor determinante en el crecimiento empresarial y desarrollo sostenible, al mostrar los aspectos en cuales se están utilizando los recursos, y los medios por cuales medir el aprovechamiento del mismo.

Con relación a la presentación de información financiera relacionada con el ámbito ambiental en los estados financieros, los casos de provisiones $y$ contingencias relacionadas con cuestiones ambientales se presenta en las notas a los estados financieros, mientras que los activos ambientales éstos se incorporan en el rubro de propiedades, planta y equipo, aunque no son identificadas como partidas de tipo ambiental. En el caso de la información de tipo cualitativa, la existencia de políticas y cumplimiento de metas de tipo ambiental no es revelada en los estados financieros.

En cuanto a la falta de revelación de las políticas y metas ambientales en las notas a los estados financieros, deja de revelar información sobre la gestión ambiental de la empresa ambiental, va en detrimento del desarrollo sustentable. Para Lozano (2016), el fomento y revelación de políticas ambientales del sector público y privado, dirigidas a un desarrollo sustentable, son de gran importancia para mantener un equilibrio empresarial, social y ambiental. El actual crecimiento económico y demográfico implica una mayor responsabilidad empresarial y de los ciudadanos, además la participación de los organismos internacionales al desarrollo ambiental se ha venido desarrollando consecutivamente en los últimos años trayendo consigo una mejor unificación socio-económica y ambiental.

En lo concerniente a los costos ambientales, estas empresas poseen ciertas categorías para las actividades de prevención, restauración, y saneamiento, incluidas dentro de los gastos operacionales sin ningún tipo de identificación del carácter ambiental de las mismas. La clasificación de estos desembolsos se corresponde con los planteamientos de Hansen y Mowen (2003) y Phalen y Fronti (2005), para quienes los costos ambientales son los resultantes de llevar a cabo las actividades voluntarias o porque existe 
una pobre calidad ambiental, así como las requeridas por contrato o las leyes para prevenir, disminuir o remediar el daño causado. Aunque, no son apropiadamente identificadas para su contabilización y presentación.

La gerencia financiera de estas empresas prepara informes periódicos sobre la existencia y consumo de materia prima, considerando de suma importancia su flujo de entrada, proceso y salida en las actividades de producción, incluyendo el tratamiento administrativocontable de los desechos para evitar la contaminación. Ese flujo, según Gray y Bebbington (2006), está basado en una concepción de sistemas referentes a las organizaciones para informar sobre las cantidades financieras y no financieras relacionadas con el uso de los recursos, permitiéndole proporcionar transparencia a la organización en cuanto a la temática ambiental.

En estas empresas la evaluación de las actividades ambientales se realiza con base en el presupuesto operativo, mediante la revisión por partidas ambientales. Las normas y procedimientos administrativos por los cuales se rigen estas organizaciones no contemplan indicadores ambientales para la evaluación de los resultados, a pesar de que, según Pahlen y Fronti (2005), el desarrollo de indicadores para la caracterización de una empresa orientada hacia el medio ambiente es un apoyo importante para la revisión y evaluación de los datos revelados acerca de su situación medioambiental.

\section{Conclusiones}

Más allá del cumplimiento de preceptos ambientales establecidos formalmente, la actividad empresarial ha de enfocar sus esfuerzos en el desarrollo sostenible. El análisis de la contabilidad de gestión ambiental en empresas del sector agroindustrial productoras de subproducto animal para la elaboración de alimentos balanceados para animales, en el municipio San Francisco del estado Zulia (Venezuela) evidencia las limitaciones importantes en el desarrollo de la misma, y que tienen impacto significativo en el desarrollo organizacional de cara al cumplimiento de su responsabilidad con el medioambiente.

La implantación de la contabilidad de gestión ambiental en las empresas contribuye a incrementar su permanencia en el tiempo, amalgamando los aspectos financieros con la gestión economía, social, y medioambiental, en una sola dirección al logro de los objetivos empresariales que contemplen su contribución hacia el desarrollo sostenible y regulación del calentamiento global.

\section{Referencias bibliográficas}

Asamblea Nacional de la República Bolivariana de Venezuela (1999), Constitución de la República Bolivariana de Venezuela. Gaceta Oficial No. 5.453 de fecha 24/03/00. Venezuela.

Asamblea Nacional de la República Bolivariana de Venezuela (2006), Ley Orgánica del Ambiente. Gaceta Oficial No. 5.833 de fecha 19/03/07. Venezuela.

Azqueta, Diego (2009), Introducción a la economía ambiental. Segunda edición, Madrid - España, Editorial Mc graw Hill.

Consejo de Normas Internacionales de Información Financiera (2011), Normas Internacionales de Información Financiera. Editado por el IASB. 
Crespo, Patricia (2005), Guía para la contabilidad medioambiental. Primera edición, España, Ediciones Gestión 2000.

Fronti, Luisa y Wainstein, Mario (2010), Contabilidad y auditoria ambiental. Primera edición Buenos Aires, Ediciones Macchi. http://www.dominicanaonline. diccionariomedioambiente. Recuperado 07-02-13.

Fundación Global Democracia y Desarrollo FUNGLODE (2013), Diccionario Enciclopédico Dominicano de Medio Ambiente. http://www.dominicanaonline. diccionariomedioambiente. Recuperado 07-02-13.

Gray, Rob y Bebbintong, Jan (2006), Contabilidad y auditoria ambiental. Segunda edición, Bogotá - Colombia, Ediciones Ecoe.

Hansen, Don y Mowen, Maryanne (2003), Administración de costos. Contabilidad y control. Tercera edición, editorial Thomson. México.

Lozano Valqui, Gerson (2016), Contabi- lidad ambiental: una herramienta empresarial para obtener el desarrollo sostenible. Revista Accounting power for business, volumen I número, de la Universidad Peruana Unión. Perú.

Mantilla, Eduardo; Vergel, Crisanto y López, José (2005), Medición de la sostenibilidad ambiental. Colombia: Editorial Universidad Cooperativa de Colombia.

Mantilla Pinilla, Eduardo; Cabeza Rozo, Mindrey Tatiana; Vargaa Barajas, Julieth Andrea (2015), La realidad del subdesarrollo y la contabilidad ambiental. Revista Saber, ciencia y libertad. Vol. 10, №. 2, de la Universidad Libre de Cartagena. Colombia.

Pahlen, Ricardo y Fronti, Luisa (2005), Contabilidad social y ambiental. Primera edición, Argentina - Buenos Aires, Ediciones Macchi.

Van, Bart; Monroy Nestor y Saer, Alex (2011), Producción más limpia. Paradigma de gestión ambiental. Bogotá, Colombia: Editorial Alfaomega Colombiana, S.A.

- Esta obra está bajo una licencia de Creative Commons Reconocimiento-NoComercialCompartirlgual 3.0 Unported.

http://creativecommons.org/licenses/by-nc-sa/3.0/deed.es_ES 